\title{
Characterization and optimization of a novel protein refolding methodology
}

\author{
G. Roussel ${ }^{1}$, S.L. Rouse ${ }^{2}$, M. Sansom ${ }^{2}$, E.A. Perpète ${ }^{1}$, and C. Michaux ${ }^{1}$ \\ ${ }^{1}$ Unité de Chimie Physique Théorique et Structurale, Université de Namur, Namur, Belgique \\ ${ }^{2}$ Structural Bioinformatics and Computational Biochemistry Unit, University of Oxford, Oxford, United-Kingdom
}

Controlling the refolding process of proteins is of considerable theoretical and practical interest [1, 2]. Recently, a simple yet effective method to recover refolded and active proteins has been developed, based on the association of an anionic detergent (the sodium dodecyl sulfate, SDS, known as denaturing agent) with an amphipathic diol solvent [3-5]. More precisely, it has been shown that peculiar solvents can modulate the denaturing properties of SDS and even induce the refolding from a SDS-denatured state. Up to now, this cosolvent effect has been observed on both soluble and bacterial membrane proteins. None to say, it is crucial to have a clear picture of the physicochemical and molecular basis of these refolding processes. To this end, we have used both experimental (intrinsic fluorescence and circular dichroïsm) and theoretical (molecular dynamics [6]) approaches to help understanding such intricate phenomena including the complex interplay between protein-SDS, protein-solvent and solvent-SDS interactions.

In that context, a fundamental investigation has been achieved starting from the study of the detergent/cosolvent couple and then considering the protein/detergent/cosolvent complex. In a first stage, we explore the micellization characteristics of SDS in presence (or absence) of solvents (chain length, one or several alcohols groups, position isomers, ... ). Such a study gives access to the nature and strength of the interactions between the molecules, the system stability as a function of time, and the micelles formation kinetics. Original detergent/solvent pairs can be proposed in order to improve the efficiency of our refolding protocol, as well as to sharpen our understanding of its mechanism, paving the way to the full protein (especially of tricky systems as membrane proteins) characterization.

\section{References}

1. K.A. Dill, S.B. Ozkan, T.R. Weikl, J.D. Chodera, V.A. Voelz, Current opinion in structural biology 17342 (2007)

2. J.U. Bowie, Nature 438 581(2005)
3. C. Michaux, N.C. Pomroy, G.G. Privé, Journal of molecular biology 3751477 (2008)

4. C. Michaux, J. Pouyez, J. Wouters, G.G. Privé, BMC structural biology 829 (2008)

5. J.A. Cuesta-Seijo, C. Neale, M.A. Khan, J. Moktar, C.D. Tran, R.E. Bishop, R. Pomès, G.G. Privé, Structure 181210 (2010)

6. J. Zhang, W. Li, J. Wang, M. Qin, L. Wu, Z. Yan, W. Xu, G. Zuo, W. Wang, IUBMB life 61627 (2009) 